\title{
Nun soll die Hepatitis C in Deutschland bis 2030 eliminiert werden
}

\begin{abstract}
Die Hepatitis $\mathrm{C}$ ist leicht zu diagnostizieren und heute mit einer einfachen, verträglichen und mittlerweile auch kosteneffektiven Therapie heilbar. Nun soll sie in Deutschland ausgerottet werden. Die bundesweite Kampagne „Bist Du Chris?“ möchte Risikogruppen aufklären und auffordern, sich testen zu lassen.
\end{abstract}

\begin{abstract}
Die Zahl der mit dem Hepatitis-CVirus infizierten Personen wird weltweit auf ca. 70 Millionen und in Deutschland auf ca. 250.000 geschätzt. Da die Infektion häufig ohne oder nur mit unspezifischen Symptomen verläuft, wissen viele Betroffene gar nichts davon. Der Hannoveraner Hepatologe Prof. Michael Manns, Vorstandvorsitzender der „Initiative pro Leber", schätzt die Dunkelziffer auf ca. $40 \%$.
\end{abstract}

\section{Tab. 1 Die Hepatitis C kann jeden treffen}

\section{Wer könnte eine Hepatitis $C$ haben?}

Bei diesen Personenkreisen sollte der Hausarzt HCV-Antikörper bestimmen:

\section{Personen mit erhöhten Leberwerten oder Symptomen einer Lebererkrankung}

2. Empfänger von Blutprodukten vor 1992

3. Hämodialyse-Patienten

4. Organtransplantatempfänger

5. Personen nach großem medizinischem Eingriff (unter unklaren Hygiene-Bedingungen)

6. Medizinisches Personal

\section{Sexualpartner von HCV-Infizierten}

8. Gefängnisinsassen

9. Personen mit Tätowierungen oder Piercings

10. HIV- oder HBV-Infizierte

11. Aktive oder ehemalige Drogenkonsumenten

12. Kinder HCV-positiver Mütter

\section{Wissen in der Bevölkerung über Hepatitis $C$ ist dürftig}

Die Kenntnisse über die Infektionskrankheit in der Bevölkerung sind einer repräsentativen Umfrage zufolge verbesserungsfähig. Nur eine Minderheit weiß, dass es sich um eine oft lange Zeit asymptomatische, aber zu 50-85\% chronisch verlaufende und langfristig potenziell tödliche Erkrankung handelt. Nur jedem Dritten ist bekannt, dass es keine Impfung gibt. Wenige wissen, dass die Krankheit seit Kurzem mit neuen Therapien in den meisten Fällen komplett geheilt werden kann.

Kernziel der jetzt angelaufenen „Initiative pro Leber" der Deutschen Leberstiftung, der Deutschen Leberhilfe e.V. und der Firma Gilead Sciences ist es, dass sich möglich viele Menschen mit einem erhöhten Risiko testen lassen und sich fragen: „Bin ich Chris?“ „Nur wer weiß, dass er krank ist, kann behandelt werden. Wer behandelt wird, hat eine sehr hohe Chance auf Heilung. Ein einfacher Bluttest kann Klarheit schaffen", sagt Prof. Manns. „Chris“als global häufiger Name für Männer und Frauen soll symbolisieren, dass die Infektion jeden treffen kann. Risikogruppen und damit übliche Ansteckungswege nennt Tab. 1.

Hepatitis C kann zu Zirrhose, hepatozellulärem Karzinom und einer Reihe von extrahepatischen Manifestationen führen, z. B. chronische Schmerzen, Fatigue, Neuropathie oder Diabetes. In
Deutschland sterben mindestens 1.300 Menschen an den Folgen von Hepatitis C, berichtete Prof. Claus Niederau, Direktor der Klinik für Innere Medizin am Klinikum in Oberhausen.

\section{Derzeit noch 5.500 Neuinfektionen jährlich}

Derzeit werden jährlich 25.000 Patienten in Deutschland behandelt, ca. 5.500 stecken sich pro Jahr neu an. Eine verstärkte Ansprache der Risikogruppen würde vielen Menschen die leidvolle Erkrankung ersparen und ist nach Modellanalysen auch für das Gesundheitssystem sinnvoll, so Niederau. Bis 2030 will man die Hepatitis eindämmen oder ganz eliminieren. Dazu ist es nötig, möglichst viele Patienten zu erkennen und $\mathrm{zu}$ therapieren. Gleichzeitig ist ein Präventionsprogramm notwendig, um die Zahl der Neuinfektionen zu senken.

Ein wichtiger Erfolgsfaktor ist neben der öffentlichen Aufklärung die Mitarbeit der Hausärzte: Sie sind die erste Anlaufstelle, sie können Patienten mit erhöhtem HCV-Risiko beraten, den Antikörpertest in die Wege leiten und den Patienten bei positivem Ergebnis überweisen.

\section{Dr. Dirk Einecke}

- Quelle: Auftakt-Pressekonferenz „Bist Du Chris?-Neue Bundesweite Kampagne für ein Deutschland ohne Hepatitis C"; Berlin, 30. Mai 2017 (Veranstalter: Initiative Pro Leber - Hepatitis C ist heilbar (Deutsche Leberstiftung, Deutsche Leberhilfe e.V., Gilead Sciences)

- Weitere Informationen: www.bist-du-chris.de 